\title{
NITRO DERIVATIVES AND OTHER CONSTITUENTS OF Aristolochia melastoma
}

\author{
Cláudio R. Nogueira e Lucia M. X. Lopes* \\ Instituto de Química, Universidade Estadual Paulista, CP 355, 14801-970 Araraquara - SP, Brasil
}

Recebido em 18/8/11; aceito em 26/10/11; publicado na web em 23/1/12

\begin{abstract}
A phytochemical study of Aristolochia melastoma Manso has led to the isolation and identification of 20 known compounds, including aristolochic acids, sodium aristolochates, lignan, flavonoids, and nitro phenylethyl derivatives. Their structures were established by spectroscopic analysis. The presence of thalictricoside and secoisolariciresinol dimethyl ether diacetate is reported for the first time in the Aristolochiaceae family. In addition, the presence of nitro compounds in this species is significant.
\end{abstract}

Keywords: Aristolochia melastoma; aristolochic acid; nitro phenylethyl derivative.

\section{INTRODUCTION}

The Aristolochiaceae are members of the order Piperales and consist of flowering plants, most of which are tropical or subtropical. ${ }^{1}$ This family is usually divided into two subfamilies: Asaroideae, which includes Asarum and Saruma, and Aristolochioideae, which includes Aristolochia sensu lato (s.1.) and Thottea. ${ }^{2}$ González and Stevenson ${ }^{3}$ reviewed the phylogenetic relationships of the Aristolochioideae and divided Aristolochia s.1. into four genera (Aristolochia sensu stricto, Pararistolochia, Endodeca, and Isotrema) within two subtribes. Their results were congruent with the scheme of the subtribes Aristolochiinae and Isotrematinae originally established by Huber. ${ }^{1}$ Furthermore, a taxonomic framework constructed based on chromosome number, as well as molecular and morphologic data, showed differences within Aristolochiaceae only in the recognition of their taxonomic ranks. ${ }^{2}$ Aristolochia s.l. consists of approximately 500 species. Brazilian Aristolochiaceae species have mainly been used in Brazilian traditional medicine as abortifacients, stomachics, antiophidians, antiasthmatics, and expectorants, and, recently, in slimming therapies. ${ }^{4,5}$ Phytochemical and pharmaceutical studies on the chemical constituents of the Aristolochia spp have mainly focused on aristolochic acids (AAs), which have a 3,4-methylenedioxy-10-nitrophenanthrenic-1-acid carbon skeleton. Among AAs, AA-I and AA-II are found in most Aristolochia species. ${ }^{4}$ The former is also known for its biological activities, such as antitumor, insecticidal, and nephrotoxic activities. ${ }^{6}$

Health food supplements containing aristolochic acids have been recently prohibited in several countries due to aristolochic acid nephropathy, which includes Chinese herb nephropathy and Balkan endemic nephropathy, all of which show renal impairment as a hallmark for the disease caused by the chronic ingestion of herbs containing these acids. ${ }^{7}$

Due to the complexity of herbal ingredients in general and their inherent biological variation, and since Aristolochia species used in the traditional Brazilian medicine have not been properly differentiated, it is necessary to determine their chemical constituents.

One of the estimated 100 Brazilian Aristolochia species is Aristolochia melastoma Manso, a little runner vine found in southern and southeastern Brazil. ${ }^{4}$ Our previous study on the essential oil from the stems and roots of $A$. melastoma showed that this species is rich in monoterpene hydrocarbons (92\%), and limonene, $\alpha$-pinene, and camphene are major constituents of the oils. ${ }^{8}$ A GC-MS analysis of

\footnotetext{
*e-mail: lopesx1@iq.unesp.br
}

the $\mathrm{CH}_{2} \mathrm{Cl}_{2}$ extract of the leaves showed high concentrations of $Z$ - and $E$-caryophyllene in the extract. ${ }^{9}$ As part of our continuing studies on the Aristolochiaceae family, we report here the isolation and structural elucidation of nitro derivatives, among other compounds, from stems and roots of $A$. melastoma.

\section{EXPERIMENTAL}

\section{General experimental procedures}

One-dimensional $\left({ }^{1} \mathrm{H},{ }^{13} \mathrm{C}\right.$, DEPT, and $g$ NOESY) and two-dimensional $\left({ }^{1} \mathrm{H}-{ }^{1} \mathrm{H} g \mathrm{COSY}, g\right.$ NOESY, $g \mathrm{HMQC}$, and ${ }^{1} \mathrm{H}-{ }^{13} \mathrm{C}$ $g \mathrm{HMBC}$ ) NMR experiments were performed on a Varian INOVA 500 spectrometer $(11.7 \mathrm{~T})$ at $500 \mathrm{MHz}\left({ }^{1} \mathrm{H}\right)$ and $126 \mathrm{MHz}\left({ }^{13} \mathrm{C}\right)$, using deuterated solvents $\left(\mathrm{CDCl}_{3}, \mathrm{DMSO}-d_{6}\right)(99.98 \% \mathrm{D})$ as an internal standard for ${ }^{13} \mathrm{C}$ NMR chemical shifts, and residual solvent as an internal standard for ${ }^{1} \mathrm{H}$ NMR. $\delta$ values are reported relative to TMS. Mass spectra (ESI-MS) were obtained on a Fisons Platform II, and flow injection into the electrospray source was used for LCESI-MS. IR spectra were obtained on a Perkin-Elmer 1600 FT-IR spectrometer using $\mathrm{KBr}$ discs. Optical rotations were measured on a Perkin-Elmer 341-LC polarimeter. Ultraviolet (UV) absorptions were measured on a Perkin-Elmer UV-vis Lambda 14P diode array spectrophotometer. HPLC analyses were performed using a Shimadzu liquid chromatograph (SPD-10 Avp) equipped with UV-vis and 341LC polarimeter detectors. RP-18 columns were used (Varian, C18, with a particle size of $5 \mu \mathrm{m}, 250 \times 4.6 \mathrm{~mm}$ for analytical analysis and $250 \times 20 \mathrm{~mm}$ for semi-preparative analysis), and chromatograms were acquired at 336 and $254 \mathrm{~nm}$. TLC: silica gel $60 \mathrm{PF}_{254}$. Melting points were recorded on a Microquímica MQAPF-302 melting point apparatus and are uncorrected.

\section{Plant materials}

Stems and roots of A. melastoma were collected in Araraquara city, São Paulo State, Brazil, in March 2008 and identified as A. melastoma Manso by Dr. C. Aranha and Dr. L. Capellari Jr. A voucher specimen (ESA88883) was deposited at the herbarium of the Escola Superior de Agricultura "Luiz de Queiroz", Piracicaba, SP, Brazil.

\section{Extraction and isolation}

Air-dried and powdered stems and roots of A. melastoma (220 g) were extracted at room temperature with hexane, $\mathrm{Me}_{2} \mathrm{CO}$, and $\mathrm{EtOH}$, 
successively, and the extracts were individually concentrated. The acetone extract ( $4.7 \mathrm{~g}$ ) was subjected to column chromatography (CC) over silica gel 60 (70-230 mesh, 100 g, Hex-EtOAc-MeOH, gradient). A total of 21 fractions ( $125 \mathrm{~mL}$ each) were collected and those with similar TLC profiles were combined, to give a total of 11 fractions. Fraction 1 was eluted with Hex, fractions 2-6 were eluted with Hex-EtOAc (9:1, $4: 1,7: 3,3: 2$, and $1: 1$, respectively), and fraction 7 was eluted with EtOAc. Fractions 8-10 were eluted with EtOAc-MeOH (9:1, 7:3, and $1: 1$, respectively) and fraction 11 was eluted with $\mathrm{MeOH}$. Fraction $5\left(50.7 \mathrm{mg}\right.$ ) was subjected to preparative TLC (Silica gel $60 \mathrm{PF}_{254}$, $\mathrm{Hex}-\mathrm{Me}_{2} \mathrm{CO}-\mathrm{EtOH}$ 6:4:0.5) to give 1 (2.1 mg) (Figure 1). Fraction 6 (113.4 mg) was fractionated over Sephadex LH-20 (EtOAc-propanol $1: 1)$ to give 2 contaminated by fatty acids $(3.0 \mathrm{mg}), \mathbf{3}(10.5 \mathrm{mg})$, and a mixture $(10.2 \mathrm{mg})$ of $\mathbf{4}$ and $\mathbf{5}$. Fractions $7(274.6 \mathrm{mg})$ and $8(168.0 \mathrm{mg})$ were individually washed with $\mathrm{CHCl}_{3}, \mathrm{Me}_{2} \mathrm{CO}$, and $\mathrm{H}_{2} \mathrm{O}$, successively. The acetone-soluble fraction from $7(42.8 \mathrm{mg})$ was subjected to HPLC [Varian analytical column 25 x 4,6 mm, $5 \mu \mathrm{m}$, eluted with $\mathrm{MeOH}-\mathrm{H}_{2} \mathrm{O}$ $(\mathrm{AcOH} 1,2 \%) 68: 32$, flow $=0,9 \mathrm{~mL} \mathrm{~min}^{-1}, \lambda=254 \mathrm{~nm}$ ] to give $\mathbf{6}(2.4$ $\mathrm{mg}), \mathbf{7}(3.4 \mathrm{mg}), \mathbf{8}(1.5 \mathrm{mg}), \mathbf{9}(1.8 \mathrm{mg})$, and $\mathbf{1 0}(3.0 \mathrm{mg})$. The remaining residue from fraction $7(38.8 \mathrm{mg})$ was subjected to $\mathrm{HPLC}\left(\mathrm{MeOH}-\mathrm{H}_{2} \mathrm{O}\right.$ 17:33) to give $\mathbf{1 1}(4.7 \mathrm{mg}$ ), whereas the remaining residue from fraction 8 gave $12(2.0 \mathrm{mg})$. The water-soluble fraction from fraction 8 (127.1 $\mathrm{mg}$ ) was subjected to CC (RP-18 silica gel, 230-400 mesh, $\mathrm{MeOH}-\mathrm{H}_{2} \mathrm{O}$ gradient) to give 20 subfractions of $125 \mathrm{~mL}$ each. Subfractions 2, 3, and 8 gave 13 (27.4 mg), 14 (6.8 mg), and 15 (3.5 mg), respectively. Fraction 9 (2.53 g) was subjected to CC (RP-18 silica gel, 230-400 mesh, $\mathrm{MeOH}-\mathrm{H}_{2} \mathrm{O}$, gradient) to give 25 subfractions of $125 \mathrm{~mL}$ each. Subfractions 5 and $6(15.7 \mathrm{mg})$ gave $16(6.8 \mathrm{mg})$ and $\mathbf{1 7}(1.0 \mathrm{mg})$, whereas subfraction 11 gave 18 (34.6 mg). Subfraction $20(45.2 \mathrm{mg})$ was subjected to HPLC [Varian analytical column $25 \mathrm{x} 4.6 \mathrm{~mm}, 5 \mu \mathrm{m}$, $\left(\mathrm{ACN}-\mathrm{MeOH}-\mathrm{H}_{2} \mathrm{O} 7: 10: 83\right.$, flow $\left.\left.=1.0 \mathrm{~mL} \mathrm{~min}^{-1}, \lambda=254 \mathrm{~nm}\right)\right]$ to give $\mathbf{1 9}(5.8 \mathrm{mg})$ and $\mathbf{2 0}(6.0 \mathrm{mg})$.

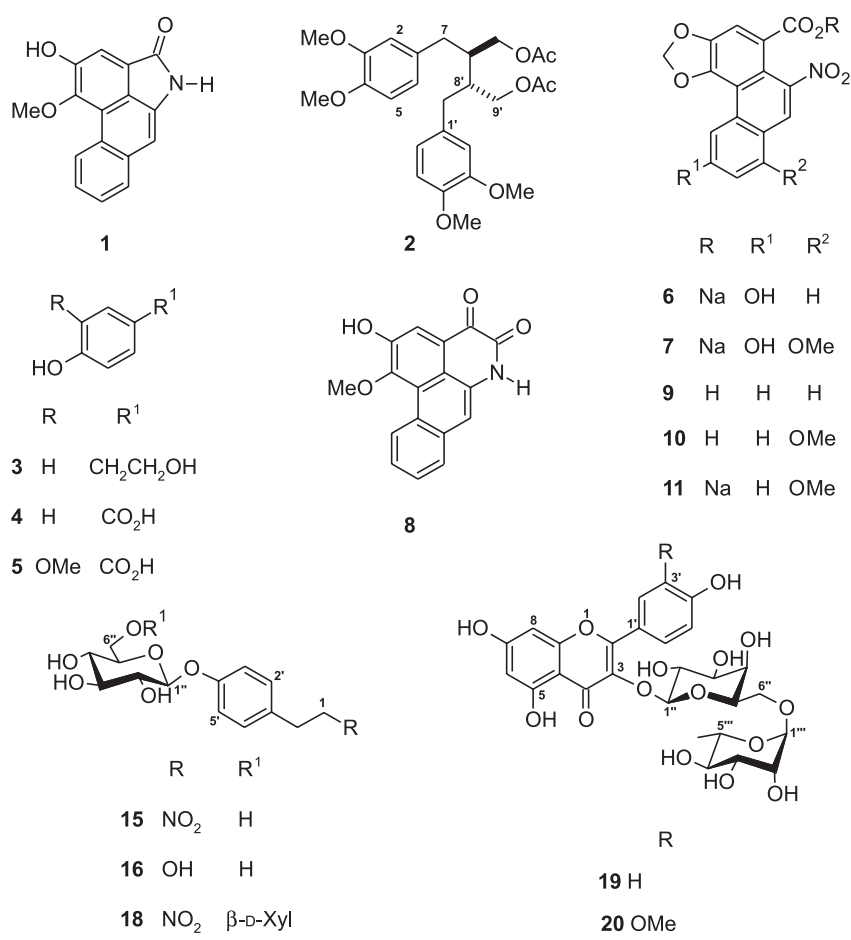

Figure 1. Chemical structures of compounds 1-11, 15, 16, and 18-20

Secoisolariciresinol dimethyl ether diacetate (2)

Positive ES-MS m/z (rel. int.) $497[\mathrm{M}+\mathrm{Na}]^{+}$(80), 295 (100); ${ }^{1} \mathrm{H}$ NMR (500 MHz, $\left.\mathrm{CDCl}_{3}\right): \delta 6.58(2 \mathrm{H}, \mathrm{d}, J=2.0 \mathrm{~Hz}, \mathrm{H}-2,2$ '), $6.78(2 \mathrm{H}, \mathrm{d}, J=8.0 \mathrm{~Hz}, \mathrm{H}-5,5$ ') $6.61(2 \mathrm{H}, \mathrm{dd}, J=8.0,2.0 \mathrm{~Hz}$,
H-6,6'), 2.67 (4H, m, H-7,7'), 2.12 (2H, m, H-8,8'), 4.05 (2H, dd, $\left.J=11.5,5.5 \mathrm{~Hz}, \mathrm{H}-9,9^{\prime}\right), 4.22$ (2H, m, H-9,9'), 3.83, (6H, s, OMe-3,3'), 3.87 (6H, s, OMe-4,4'), 2.08 (6H, s, Me); ${ }^{13} \mathrm{C}$ NMR $\left(126 \mathrm{MHz}, \mathrm{CDCl}_{3}\right): \delta 132.2\left(\mathrm{C}^{-1,1}{ }^{\prime}\right), 112.1\left(\mathrm{C}-2,2^{\prime}\right), 148.9$ (C-3,3'), 147.5 (C-4,4'), 111.2 (C-5,5'), 121.0 (C-6,6'), 34.9 (C-7,7'), 39.7 (C-8,8'), 64.4 (C-9,9'), 55.8, 55.9 (OMe-3,3',4,4'), $171.0(\mathrm{C}=\mathrm{O}), 21.0(\mathrm{Me})$.

\section{Thalictricoside (18)}

Colorless solid, m.p. $124.5-124.9{ }^{\circ} \mathrm{C}(\mathrm{MeOH}),[\alpha]_{\mathrm{D}}{ }^{29}-63.3^{\circ}$ (MeOH, c. 0.13); Positive ES-MS m/z (rel. int.) 484 [M+Na $]^{+}(100)$; ${ }^{1} \mathrm{H}$ NMR (500 MHz, DMSO- $\left.d_{6}\right): \delta 4.79(2 \mathrm{H}, \mathrm{t}, J=7.0, \mathrm{H}-1), 3.17(2 \mathrm{H}$, t, $J=7.0 \mathrm{~Hz}, \mathrm{H}-2), 7.18$ (2H, d, $\left.J=8,8 \mathrm{~Hz}, \mathrm{H}-2^{\prime}, 6^{\prime}\right), 7.00$ (2H, d, $J$ $=8.8 \mathrm{~Hz}, \mathrm{H}-3$ ',5'), 4.78 (1H, d, J = 7.5, H-1'”), 3.23 (1H, m, H-2”), 3.25 (1H, m, H-3"), 3.16 ( $1 \mathrm{H}, \mathrm{dd}, W_{1 / 2}=9.5,8.5 \mathrm{~Hz}, \mathrm{H}-4$ ") $), 3.51$ $\left(1 \mathrm{H}, \mathrm{ddd}, W_{1 / 2}=9.5,6.5,1.4 \mathrm{~Hz}, \mathrm{H}-5 "\right), 3.95(1 \mathrm{H}, \mathrm{dd}, J=11.4,1.4$ Hz, H-6”), 3.57 (1H, dd, $W_{1 / 2}=11.4,6.5 \mathrm{~Hz}, \mathrm{H}-6$ "), 4.18 (1H, d, J $=7.7 \mathrm{~Hz}, \mathrm{H}-1$ " ") $) 2.98\left(1 \mathrm{H}, \mathrm{dd}, W_{1 / 2}=8.8,7.7 \mathrm{~Hz}, \mathrm{H}-2\right.$ "' $), 3.08(1 \mathrm{H}$, t, $W_{1 / 2}=8.8 \mathrm{~Hz}, \mathrm{H}-3$ "' $), 3.27$ (1H, m, H-4"”), 2.94 (1H, dd, $J=11.3$, $10.3 \mathrm{~Hz}, \mathrm{H}-5$ "'), 3.67 (1H, dd, $J=11.3,5.3 \mathrm{~Hz}, \mathrm{H}-5$ "'); ${ }^{13} \mathrm{C}$ NMR $\left(126 \mathrm{MHz}, \mathrm{DMSO}-d_{6}\right)$ : $\delta 76.2$ (C-1), 31.7 (C-2), 129.6 (C-1'), 129.6 (C-2',6'), 116.4 (C-3',5'), 156.3 (C-4'), 100.5 (C-1'), 73.2 (C-2"'), 76.4 (C-3"), 69.6, 69.5 (C-4",4"'), 75.8 (C-5"), 68.1 (C-6”), 103.8 (C-1"'), 73.3 (C-2"'), 76.4 (C-3"'), 65.6 (C-5"').

\section{Kaempferol 3-O- $\beta$-robinobioside (19)}

Colorless solid, $[\alpha]_{\mathrm{D}}{ }^{29}-29.5^{\circ}(\mathrm{MeOH}$, c. 0.04); Negative ES-MS $\mathrm{m} / \mathrm{z}$ (rel. int.) $593[\mathrm{M}-\mathrm{H}]^{-}(100) ;{ }^{1} \mathrm{H} \mathrm{NMR}\left(500 \mathrm{MHz}, \mathrm{CD}_{3} \mathrm{OD}\right): \delta$ $6.22(1 \mathrm{H}, \mathrm{d}, J=1.9 \mathrm{~Hz}, \mathrm{H}-6), 6.41(1 \mathrm{H}, \mathrm{d}, J=1.9 \mathrm{~Hz}, \mathrm{H}-8), 8.09$ (2H, d, $J=9.0 \mathrm{~Hz}, \mathrm{H}-2^{\prime}, 6$ ' $), 6.89$ (2H, d, $J=9.0 \mathrm{~Hz}, \mathrm{H}-3$ ' , 5'), 5.04 $(1 \mathrm{H}, \mathrm{d}, J=8.0 \mathrm{~Hz}, \mathrm{H}-1$ ") 3.79 (1H, dd, $J=9.8,8.0 \mathrm{~Hz}, \mathrm{H}-2$ "), 3.54 (1H, dd, $J=9.8,3.3 \mathrm{~Hz}, \mathrm{H}-3$ ") $), 3.77$ (1H, dd, $J=3.3,0.9 \mathrm{~Hz}$, H-4"), 3.62 (1H, ddd, $J=7.0,5.5,0.9 \mathrm{~Hz}, \mathrm{H}-5$ "), 3.40 (1H, dd, $J=10.5,7.0 \mathrm{~Hz}, \mathrm{H}-6$ "), 3.73 (1H, dd, $J=10.5,5.5 \mathrm{~Hz}, \mathrm{H}-6$ "), 4.52 (1H, d, $\left.J=1.5 \mathrm{~Hz}, \mathrm{H}-1{ }^{\prime \prime} "\right), 3.59$ (1H, dd, $J=3.3,1.5 \mathrm{~Hz}, \mathrm{H}-2$ '”'), $3.50\left(1 \mathrm{H}, \mathrm{dd}, J=9.3,3.3 \mathrm{~Hz}, \mathrm{H}-3{ }^{\prime \prime} "\right), 3.27(1 \mathrm{H}, \mathrm{t}, J=9.3 \mathrm{~Hz}$, H-4"'), 3.53 (1H, m, H-5"'), 1.18 (3H, d, J=6.5 Hz, H-6" '); ${ }^{13} \mathrm{C}$ NMR (126 MHz, CD $\mathrm{OD}): \delta 159.4$ (C-2), 135.7 (C-3), 179.6 (C4), 163.0 (C-5), 100.0 (C-6), 166.3 (C-7), 94.9 (C-8), 158.6 (C-9), 105.6 (C-10), 122.7 (C-1'), 132.4 (C-2',6'), 116.1 (C-3',5'), 161.0 (C-4'), 105.6 (C-1"), 73.0 (C-2"), 75.1 (C-3"), 70.2 (C-4"), 75.4 (C-5"), 67.5 (C-6"), 102.0 (C-1"”), 72.1 (C-2"'), 72.3 (C-3"'), 73.9 (C-4"'), 69.7 (C-5"'), 18.0 (C-6"').

\section{Isorhamnetin 3-O- $\beta$-robinobioside (20)}

Yellow solid, $[\alpha]_{\mathrm{D}}{ }^{29}-25.5^{\circ}(\mathrm{MeOH}$, c. 0.05); Negative ES-MS $\mathrm{m} / \mathrm{z}$ (rel. int.) $623[\mathrm{M}-\mathrm{H}]^{-}(100) .{ }^{1} \mathrm{H} \mathrm{NMR}\left(500 \mathrm{MHz}, \mathrm{CD}_{3} \mathrm{OD}\right): \delta$ $3.97(3 \mathrm{H}, \mathrm{s}, \mathrm{OMe}), \delta 6.22(1 \mathrm{H}, \mathrm{d}, J=1.7 \mathrm{~Hz}, \mathrm{H}-6), 6.42(1 \mathrm{H}, \mathrm{d}$, $J=1.7 \mathrm{~Hz}, \mathrm{H}-8), 8.03\left(1 \mathrm{H}, \mathrm{d}, J=2.0 \mathrm{~Hz}, \mathrm{H}-2^{\prime}\right), 6.91(1 \mathrm{H}, \mathrm{d}, J=$ $8.5 \mathrm{~Hz}, \mathrm{H}-5$ '), 7.61 (1H, dd, $J=8.5,2.0 \mathrm{~Hz}, \mathrm{H}-6$ '), $5.22(1 \mathrm{H}, \mathrm{d}$, $J=7.5 \mathrm{~Hz}, \mathrm{H}-1$ "), 3.82 (1H, dd, $J=9.3,7.5 \mathrm{~Hz}, \mathrm{H}-2$ "), 3.55 (1H, dd, $J=9.3,3.5 \mathrm{~Hz} \mathrm{H}-3$ "), 3.79 (1H, br d, $J=3.5 \mathrm{~Hz}, \mathrm{H}-4$ "), 3.66 (1H, m, H-5"), 3.47 (1H, dd, $J=10.5,7.0 \mathrm{~Hz}, \mathrm{H}-6 "), 3.74(1 \mathrm{H}$, dd, $J=10.5,5.5 \mathrm{~Hz}, \mathrm{H}-6$ "), 4.53 (1H, d, $J=1.5 \mathrm{~Hz}, \mathrm{H}-1$ '”), 3.58 (1H, m, H-2"'), 3.49 (1H, dd, $J=9.5,3.4 \mathrm{~Hz}, \mathrm{H}-3$ "' '), 3.27 (1H, t, $J=9.5 \mathrm{~Hz}, \mathrm{H}-4$ "' $), 3.53$ (1H, m, H-5"'), 1.18 (3H, d, $J=6.3$ $\mathrm{Hz}, \mathrm{H}-6 " ')$ ); ${ }^{13} \mathrm{C}$ NMR (126 MHz, CD $\left.\mathrm{OD}\right): \delta 57.0$ (OMe), 158.9 (C-2), 135.5 (C-3), 179.5 (C-4), 163.1 (C-5), 100.0 (C-6), 166.2 (C-7), 94.9 (C-8), 158.5 (C-9), 105.7 (C-10), 123.0 (C-1'), 114.7 (C-2'), 148.4 (C-3'), 150.9 (C-4'), 116.0 (C-5'), 123.8 (C-6'), 105.0 (C-1"), 73.1 (C-2"'), 75.1 (C-3"), 70.1 (C-4"), 75.6 (C-5"), 67.5 (C-6"), 102.0 (C-1"'), 72.1 (C-2"”), 72.3 (C-3"'), 73.9 (C-4"”), 69.8 (C-5"'), 18.0 (C-6"”). 


\section{RESULTS AND DISCUSSION}

Compounds 1 and 3-20 (Figure 1) were identified as aristolactam AII (1), ${ }^{10}$ tyrosol (3), ${ }^{11} p$-hydroxybenzoic acid (4), ${ }^{12}$ vanillic acid (5), ${ }^{13}$ sodium aristolochate IIIa (6), sodium aristolochate IVa (7), ${ }^{14}$ 4,5-dioxodehydroasimilobine (8), ${ }^{15}$ aristolochic acid II (9), aristolochic acid I (10), sodium aristolochate I (11) ${ }^{14}$ sitosterol-3-O$\beta$-glucopyranoside (12), ${ }^{11,16}$ glycerol (13), ${ }^{17}$ uridine (14), ${ }^{18}$ thalictoside (15), ${ }^{19}$ icariside D2 (16), ${ }^{20}$ adenosine $(\mathbf{1 7}),{ }^{21}$ thalictricoside $(\mathbf{1 8}),{ }^{22}$ kaempferol 3-O- $\beta$-robinobioside (19), ${ }^{23}$ and isorhamnetin 3-O- $\beta$ robinobioside $(\mathbf{2 0})^{24}$ based on analyses of their MS, ${ }^{1} \mathrm{H}$ and ${ }^{13} \mathrm{C}$ NMR (including DEPT, $g$ NOESY, ${ }^{1} \mathrm{H}-{ }^{1} \mathrm{H} g \mathrm{COSY}, g \mathrm{HMQC}, g \mathrm{HMBC}$, and $g$ NOESY) spectra and on comparisons of their spectrometric data with those reported in the literature. The substituent positions in the structures of these compounds were determined with the assistance of $g \mathrm{NOESY}$ and $g \mathrm{HMBC}$ experiments. Based on the results of these 1D- and 2D-NMR techniques, the complete assignments of NMR data obtained for hydrogens and carbons of compounds $\mathbf{1 9}$ and $\mathbf{2 0}$, including the hydrogen multiplicities, are given in the Materials and Methods section. Furthermore, the data obtained for $\mathbf{2 0}$ evidenced that the chemical shifts previously assigned ${ }^{24}$ for C-3" and C-2"' should be interchange.

${ }^{1} \mathrm{H}$ and ${ }^{13} \mathrm{C}$ NMR data obtained for $\mathbf{2}$ were consistent with those previously reported for secoisolariciresinol dimethyl ether diacetate. ${ }^{25}$ However, gHMBC experiments showed correlations between C-3,3' $\left(\delta_{\mathrm{C}} 148.9\right)$ and $\mathrm{H}-5,5$ ' $\left(\delta_{\mathrm{H}} 6.78\right)$, as well as between $\mathrm{C}-4,4$ ' $\left(\delta_{\mathrm{C}} 147.5\right)$ and $\mathrm{H}-2,2^{\prime}\left(\delta_{\mathrm{H}} 6.58\right)$ and $\mathrm{H}-6,6,\left(\delta_{\mathrm{H}} 6.61\right)$, which led to an interchange of the carbon chemical shift assignments in relation to those previously reported.

The present results show that $A$. melastoma is a source of several nitro compounds such as $\mathbf{6 - 9}, \mathbf{1 1}, \mathbf{1 5}$, and 18. Aristolochic acids have been found among the Aristolochiaceae species, as well as in the butterflies (Lepidoptera belonging to the tribes Troidine and Zerynthiini) that feed on such plants, ${ }^{26}$ whereas the nitro phenylethyl derivatives occur in plant species belonging to the orders Ranunculales and Alismatales, as well as in species within Magnoliids. The significant occurrence of nitro derivatives in this species suggests that the Aristolochiaceae family may have evolved toward nitro compound biosyntheses. Thus, further studies are needed on the safety of Brazilian Aristolochia spp.

\section{ACKNOWLEDGEMENTS}

The authors thank Dr. C. Aranha and Dr. L. Capellari Jr. for plant identification. We also thank the Fundação de Amparo à Pesquisa do Estado de São Paulo (FAPESP) and Conselho Nacional de Desenvolvimento Científico e Tecnológico (CNPq) for financial support and fellowships.

\section{REFERENCES}

1. Wanke, S.; González, F.; Neinhuis, C.; Int. J. Plant Sci. 2006, 167, 1215.

2. Berjano, R.; Roa, F.; Talavera, S.; Guerra, M.; Plant Syst. Evol. 2009, $280,219$.

3. González, F.; Stevenson, D. W.; Rev. Acad. Colomb. Cienc. Exactas Fis. Nat. 2002, 26, 25.
4. Lopes, L. M. X.; Nascimento, I. R.; da Silva, T. Em Research Advances in Phytochemistry; Mohan, R. M. M., ed.; Global Research Network: Kerala, 2001, vol. 2, p. 19-108.

5. Machado, M. B.; Lopes, L. M. X.; Phytochemistry 2008, 69, 3095.

6. Houghton, P. J.; Ogutveren, M.; Phytochemistry 1991, 30, 253; Kumar, V.; Poonam, Prasad, A. K.; Parmar, V. S.; Nat. Prod. Rep. 2003, 20, 565; Nascimento, I. R.; Murata, A. T.; Bortoli, S. A. de; Lopes, L. M. X.; Pest Manag. Sci. 2004, 60, 413; Shibutani, S.; Bonala, R. R.; Rosenquist, T.; Rieger, R.; Suzuki, N.; Johnson, F.; Miller, F.; Grollman, A. P.; Int. J. Cancer 2010, 127, 1021.

7. Chen, T.; J. Food Drug Anal. 2007, 15, 387; Martena, M. J.; Wielen, J. C. A.; Laak, L. F. J.; Konings, E. J. M.; Groot, H. N.; Rietjens, I. M. C. M.; Anal. Bioanal. Chem. 2007, 389, 263; Shaohua, Z.; Ananda, S.; Ruxia, Y.; Liang, R.; Xiaorui, C., Liang, L.; Forensic Sci. Int. 2010, 199, e5.

8. Francisco, C. S.; Messiano, G. B.; Lopes, L. M. X.; Tininis, A. G.; Oliveira, J. E. de; Capellari, Jr. L.; Phytochemistry 2008, 69, 168.

9. Silva-Brandão, K. L.; Solferini, V. N.; Trigo, J. R.; Biochem. Syst. Ecol. 2006, 34, 291

10. Tsuruta, A. Y.; Bomm, M. D.; Lopes, M. N.; Lopes, L. M. X.; Eclet. Quim. 2002, 27, 103.

11. Navickiene, H. M. D.; Lopes, L. M. X.; J. Braz. Chem. Soc. 2001, 12, 467.

12. O'Connor, C. J.; Mclennan, D. J.; Calvert, D. J.; Mitha, A. S. H.; Aust. J. Chem. 1987, 40, 677; Zhou, Y.; Gao, G.; Li, H.; Qu, J.; Tetrahedron Lett. 2008, 49, 3260.

13. González-Baró, A. C.; Parajón-Costa, B. S.; Franca, C. A.; Pis-Diez, R.; J. Mol. Struct. 2008, 889, 204.

14. Chiang, C.-Y.; Leu, Y.-L.; Chan, Y.-Y.; Wu, T.-S.; J. Chin. Chem. Soc. 1998, 45, 93; Leu, Y.-L.; Chan, Y.-Y.; Wu, T.-S.; Phytochemistry 1998, 48, 743; Nascimento, I. R.; Lopes, L. M. X.; Phytochemistry 2003, 63, 953.

15. Urzúa, A.; Freyer, A. J.; Shamma, M.; J. Nat. Prod. 1987, 50, 305.

16. Faizi, S.; Ali, M.; Saleem, R.; Irfanullah; Bibi. S.; Magn. Reson. Chem. 2001, 39, 399.

17. Breitmaier, E.; Structure elucidation by NMR in organic chemistry, John Wiley \& Sons: Chichester, 2002.

18. Saito, Y.; Nyilas, A.; Agrofoglio, L. A.; Carbohydr. Res. 2001, 331, 83.

19. Ina, H.; Iida, H.; Chem. Pharm. Bull. 1986, 34, 726.

20. Peng, Y.; Luo, J.; Lu, Q.; Chen, X.; Xie, Y.; Chen, L.; Yang, W.; Du, S.; J. Pharm. Biomed. Anal. 2009, 49, 828.

21. Ciuffreda, P.; Casati, S.; Manzocchi, A.; Magn. Reson. Chem. 2007, 45, 781.

22. Erdemgil, F. Z.; Baser, K. H. C.; Akbay, P.; Sticher, O.; Çalis, I.; Z. Naturforsch. 2003, 58C, 632.

23. Hou, W.-C.; Lin, R.-D.; Lee, T.-H.; Huang, Y.-H.; Hsu, F.-L.; Lee, M.H.; J. Sci. Food Agric. 2005, 85, 615; Nahrstedt, A.; Hungeling, M.; Petereit, F.; Fitoterapia 2006, 77, 484.

24. Komissarenko, N. F.; Chernobai, V. T.; Kolesnikov, D. G.; Chem. Pharm. Res. Inst. 1964, 158, 904; Mbark, A. N.; Charrouf, Z.; Guillaume, D.; Kol, O.In Studies in Plant Science (Advances in Plant Glycosides, Chemistry and Biology), Elsevier Science B.V.: Amsterdam, 1999, vol. 6, p. 314-319.

25. Chen, C.-C.; Hsin, W.-C.; Huang, Y.-L.; J. Nat. Prod. 1998, 61, 227.

26. Sime, K. R.; Feeny, P. P.; Haribal, M. M.; Chemoecology 2000, 10, 169. 